\title{
Acknowledgment to Reviewers of Magnetochemistry in 2020
}

\author{
Magnetochemistry Editorial Office \\ MDPI AG, St. Alban-Anlage 66, 4052 Basel, Switzerland
}

Citation: Magnetochemistry Editorial Office. Acknowledgment to Reviewers of Magnetochemistry in 2020. Magnetochemistry 2021, 7, 17. https://doi.org/10.3390/

magnetochemistry7020017

Published: 21 January 2021

Publisher's Note: MDPI stays neutral with regard to jurisdictional claims in published maps and institutional affiliations.

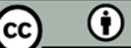

Copyright: $\odot 2021$ by the author. Licensee MDPI, Basel, Switzerland. This article is an open access article distributed under the terms and conditions of the Creative Commons Attribution (CC BY) license (http://creativecommons.org/licenses/by/4.0/).

Peer review is the driving force of journal development, and reviewers are gatekeepers who ensure that Magnetochemistry maintains its standards for the high quality of its published papers. Thanks to the cooperation of our reviewers, in 2020, the median time to first decision was 13 days and the median time to publication was 31 days. The editors would like to express their sincere gratitude to the following reviewers for their precious time and dedication, regardless of whether the papers were finally published:

A. Ekanger, Levi

Abramov, Pavel A.

Ajejas, Fernando

Al-Obaidi, Hisham

Andruh, Marius

Angelovski, Goran

Aperis, Alexandros

Arion, Vladimir

Arnaboldi, Serena

Aronica, Laura Antonella

Baddorf, Arthur P.

Balachandran, Vinitha

Balogh-Weiser, Diana

Bandyopadhyay, Supriyo

Barwiolek, Magdalena

Bazhina, Evgeniya

Beković, Miloš

Béreau, Virginie

Bernand-Mantel, Anne

Bessais, Lotfi

Blazek, Joseb

Bogomyakov, Artem

Boujtita, Mohammed

Bronisz, Robert

Brown, Katherine

Brusso, Jaclyn L.

Burzo, Emil

Cai, Kai

Cao, Jian

Catalano, Enrico

Cavallini, Massimiliano

Chorazy, Szymon

Chwastek, Krzysztof

Clérac, Rodolphe

Coisson, Marco

Cojocaru, Florina
Colacio, Enrique

Coppel, Yannick

Cornia, Andrea

Corte, Hector

D'Orazio, Franco

Da Silva Candal, Andrés

Das, Bhaskar

Dayen, Jean François

De Graaf, Coen

Deriszadeh, Ali

Diehl, Bernd

Douvalis, Alexios P.

Dreiser, Jan

Duan, Yan

Dubin, Grzegorz

Efimov, Nikolay N.

Elsaady, Wael

Ernesto-Casanova, Oscar

Etcheverry-Berrios, Alvaro

Eykyn, Thomas

Farach-Carson, Mary C.

Favieres, Cristina

Fedin, Matvey

Feiguin, Adrian

Ferbinteanu, Marilena

Fernandes, Tiago A.

Ferrauto, Giuseppe

Filice, Marco

Filipecki, Jacek

Fiorillo, Fausto

Fitta, Magdalena

Fontanesi, Claudio

Fraga-García, Paula

Gale, Eric

Gallo Cordova, Alvaro

Garbacz, Piotr 
Garcia-Sanchez, Felipe

Gębara, Piotr

Geraldes, Carlos

Gerothanassis, Ioannis P.

Gjokas, Margaritis

Gomes, Clara S. B.

Gondro, Joanna

Gornakov, Vladimir

Gravel, Edmond

Grée, René

Guillou, Francois

Guo, Zhan-Hu

Han, Ke

Hay, Moya Anne

Herrero, Roberto

Hoshino, Norihisa

Hsu, Shang-Te Danny

Huber, Gaspard

Huple, Deepak

Ishikawa, Ryuta

Janutka, Andrzej

Józefczak, Arkadiusz

Jung, Julie Cathy Antoinette Odile

Kadnár, Milan

Kajiwara, Takashi

Karadakov, Peter

Katoh, Keiichi

Kern, Frank

Kertmen, Ahmet

Kim, Ji-Hun

Kim, Jung Kyu

Kim, Nam-Young

Kiskin, Mikhail

Koizumi, Hiroyasu

Konieczny, Piotr

Korchagin, Denis V.

Korshunov, Maxim M.

Korzhikov-Vlakh, Viktor

Kosyanov, Denis Yu.

Krauklis, Andrey E.

Kriener, Markus

Kruk, Andrzej

Kubo, Atsushi

Kuchi, Rambabu

Kühne, Irina A.

Kumal, Raju

Kumar, Raj

Kuntsevich, Alexander Yu

Kuppusamy, Senthil Kumar

Lada, Zoi G.

Lahlil, Rachid
Lamichhane, Tej

Law, Jia Yan

Lemcke, Heicko

Lezama, Luis

Li, Wenlu

Li, Ying

Linert, Wolfgang

Liu, Xinyu

López-Cabrelles, Javier

Łukowski, Paweł

Lunghi, Alessandro

Lutter, Jacob C.

Luty, Tadeusz

Maarouf, Ahmed

Maesato, Mitsuhiko

Mameli, Valentina

Mameri, Samir

Mammeri, Fayna

Matsuda, Yasuhiro H.

Maxim, Cătălin

Mcgaughey, Alan

Meersmann, Thomas

Menichetti, Luca

Meyer, Franc

Meyer, Klas

Miikkulainen, Ville

Mikuriya, Masahiro

Miletić, Goran I.

Miralles, Sara G.

Misztalewska-Turkowicz, Iwona

Mondal, Amit Kumar

Moos, Torben

Moreno Pineda, Eufemio

Moskvin, Alexander S.

Müller, Danny

Murray, Keith

Nakonieczny, Damian

Navarro-Quezada, Andrea

Need, Ryan

Newton, Graham N.

Nishinaga, Tohru

Novikov, Valentin

Ogawa, Akiya

Onchoke, Kefa

Orlenko, Elena

Oshima, Yugo

Palyi, Gyula

Panzarasa, Guido

Patrulea, Viorica

Paulsen, Hauke

Pelka, Robert 
Pellico, Juan

Peralta, Juan E.

Pereiro, Rosario

Perfetti, Mauro

Pichon, Céline

Ploetz, Evelyn

Podgajny, Robert

Poggini, Lorenzo

Potapov, Andrei

Prat, Maria

Rakhmatullin, Aydar

Ranjbar, Navid

Ravera, Enrico

Reta, Daniel

Ricart, Susagna

Riegel, Harald

Rigamonti, Luca

Rikken, Geert

Rodrigues, Davi R.

Rousset, Elodie

Šalitroš, Ivan

Santana, Vinicius Tadeu

Santini, Paolo

Sarafidis, Charalampos S.

Sava, Bogdan A.

Schwaminger, Sebastian

Shepelytskyi, Yurii

Sherry, A. Dean

Shvartsman, Vladimir

Signore, Giovanni

Simeonidis, Konstantinos

Soave, Raffaella

Speliotis, Thanassis

Stefanczyk, Olaf

Stergar, Janja

Storsberg, Joachim

Stückler, Martin

Tagawa, Toshio

Temst, Kristiaan
Terasaki, Ichiro

Tesi, Lorenzo

Tetean, Romulus

Thibault, Christophe

Titma, Tiina

Tobrman, Tomáš

Tomašovičová, Natália

Tretyakov, Evgeny V.

Tsukerblat, Boris

Valladares, Luis De Los Santos

Váradi, Csaba

Varma, Vijaykumar B.

Vasko, Petra

Vejpravová, Jana Kalbacova

Vergara, José

Verma, Pragya

Vincente, Ramon

Voda, Raluca

Von Bardeleben, H.J.

Wang, Faxing

Watts, Bernard Enrico

Weber, Daniel

Wojtkielewicz, Agnieszka

Wolny, Juliusz A.

$\mathrm{Xu}$, Shoujun

Yamase, Hiroyuki

Yasuhara, Ryo

Zaleski, Curtis M.

Zarifi, Mohammad H.

Zarzycki, Arkadiusz

Zhang, Ruiqi

Zhang, Xin

Zhang, Yunyan

Zicari, Giorgio

Zorina, Leokadiya V.

Zubarev, Andrey

Zverev, Vladimir I.

Żywczak, Antoni 